\title{
Analysis of Dither in Relay Feedback Systems ${ }^{1}$
}

\section{Luigi Iannelli ${ }^{2}$}

\author{
Ulf Jönsson ${ }^{4}$
}

Francesco Vasca ${ }^{5}$

\begin{abstract}
Dither signals provide an effective way of compensating for nonlinearities in control systems. The seminal works by Zames and Shneydor and more recently by Mossaheb present rigorous tools for systematic design of dithered systems. Their results rely however on a Lipschitz assumption on the nonlinearity and thus do not cover important applications with discontinuities. This paper presents initial results on how to analyze and design dither in nonsmooth systems. In particular, it is shown that a dithered relay feedback system can be approximated by a smoothed system obtained through an averaging approach. Guidelines are given for tuning the amplitude and the period time of the dither signal, in order to stabilize the nonsmooth system.
\end{abstract}

\section{Introduction}

The use of dither signals for stabilization of nonlinear control systems is a well-known and frequently used technique. The idea is that by injecting a suitably chosen highfrequency signal in the control loop, the nonlinear sector is effectively narrowed and the system can thereby be stabilized. Theoretical justification of this idea for systems with continuous nonlinearities has been obtained by Zames and Shneydor [1, 2] and Mossaheb [3]. Their results rely however on a crucial Lipschitz assumption on the nonlinearity and thus do not cover important applications with discontinuities. Indeed, discontinuous nonlinearities in feedback control systems with high-frequency excitations appear in a large variety of models, including systems with adaptive control [4], friction [5, 6], pulse-width modulated converters [7], quantizers [8], relays [9], and variable-structure controllers [10]. In their paper on the analysis of the (smooth) LuGre friction model, Pervozvanski and Canudas de Wit [11] point out that a rigorous analysis of dither in discontinuous systems does not exist. Dither

\footnotetext{
${ }^{1}$ The work by K. H. Johansson and U. Jönsson was supported by the Swedish Research Council.

${ }^{2}$ Dipartimento di Informatica e Sistemistica, Università di Napoli Federico II, Napoli, Italy, e-mail: luiannel@unina.it

${ }^{3}$ Department of Signals, Sensors \& Systems, Royal Institute of Technology, Stockholm, Sweden e-mail: kallej@s3.kth.se

${ }^{4}$ Department of Mathematics, Royal Institute of Technology, Stockholm, Sweden e-mail: ulfjemath.kth. se

${ }^{5}$ Dipartimento di Ingegneria, Università del Sannio, Benevento, Italy e-mail: vasca@unisannio.it
}

tuning in nonsmooth systems is to our knowledge limited to approximate design methods mainly based on describing functions [12].

The contribution of the paper is to present an initial attempt to develop a theory for the design of dither in nonsmooth systems. We limit the analysis to an important class of nonlinearities and dither signals, namely, relay systems with triangular dither signals. Our main result states that a dithered relay feedback system can be approximated by a system with no dither and the relay replaced by a saturation. The dither period determines the accuracy of the approximation. The dither amplitude determines the gain of the saturation and thus the stability of the smoothed system. Exponential stability of the smoothed system is linked to practical stability of the dithered system through a theorem based on a frequency response criterion similar to the circle and Popov criteria. The theoretical results suggest a procedure for tuning dither systems.

In spite of the particular class of systems considered, our results can be applied to several control applications. For example some PWM power electronic converters can be represented as relay feedback systems with dither. Averaging techniques are routinely used for design of such systems and some rigorous results have been developed in $[13,14]$.

The outline of the paper is as follows. Some notation is introduced in Section 2. A motivating example is presented in Section 3, illustrating how a high-frequency dither signal can be injected to dissolve oscillations in relay feedback systems. The main theorem is presented in Section 4 and it states that the solutions of the dithered system can be arbitrarily well approximated by the solutions of a smoothed system. The section also discusses practical stability. Section 5 relates these results to dither design, which is applied to the example. The paper is concluded in Section 6, where topics for future work is discussed.

\section{Preliminaries}

The dithered system is a relay feedback system (see Figure 1)

$$
\dot{x}(t)=L x(t)+b n(c x(t)+\delta(t)), \quad x(0)=x_{0} .
$$

Here $L, b$, and $c$ are constant matrices of dimensions $q \times q$, $q \times 1$, and $1 \times q$, respectively, $q>0$. The nonlinearity $n$ : 


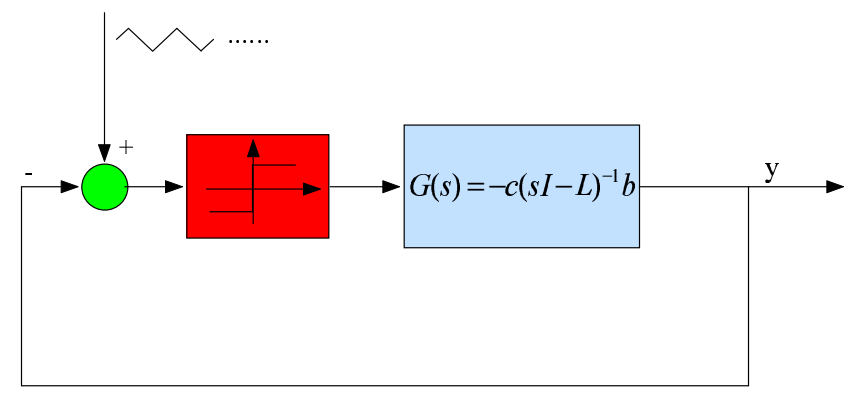

Figure 1: Dithered system.

$\mathbb{R} \rightarrow \mathbb{R}$ is given by the relay characteristic

$$
n(z)=\operatorname{sgn}(z)= \begin{cases}1, & z>0 \\ 0, & z=0 \\ -1, & z<0 .\end{cases}
$$

The dither signal $\delta:[0, \infty) \rightarrow \mathbb{R}$ is a triangle wave of amplitude $A>0$ and period $p>0$, i.e., $\delta(t+p)=\delta(t)$ for all $t$ and

$$
\delta(t)= \begin{cases}\frac{4 A}{p} t, & t \in[0, p / 4) \\ -\frac{4 A}{p} t+2 A, & t \in[p / 4,3 p / 4) \\ \frac{4 A}{p} t-4 A, & t \in[3 p / 4, p) .\end{cases}
$$

Throughout the paper we assume that the relay feedback system has a solution $x:[0, \infty) \rightarrow \mathbb{R}^{n}$ (in a classical sense), which on every compact subinterval of $[0, \infty)$ is $C^{1}$ everywhere except at finitely many points. We sometimes use the notation $x\left(t, x_{0}\right)$ for the solution of (1).

The smoothed system is given by

$$
\dot{w}(t)=L w(t)+b N(c w(t)), \quad w(0)=w_{0},
$$

where the smoothed nonlinearity $N: \mathbb{R} \rightarrow \mathbb{R}$ is defined as the average $N(z)=p^{-1} \int_{0}^{p} n(z+\delta(t)) d t$. For the relay, it is easy to show that

$$
N(z)=\operatorname{sat}(z / A)= \begin{cases}1, & z>A \\ z / A, & |z|<A \\ -1, & z<-A .\end{cases}
$$

It will be shown below that the smoothed system in many cases is a good approximation of the dithered relay feedback system. Therefore analysis and design can be performed on the smoothed system, which is often easier to treat, and then be carried over to the dithered system.

Note that the term "smoothed system" is standard in the literature on dither design and refer to that the nonlinear sector is narrowed by the dither signal. The nonlinearity is not necessarily $C^{\infty}$, as illustrated above by the saturation function being the smoothed nonlinearity corresponding to the sign function.

We use $|\cdot|$ to denote the Euclidean norm of a vector and $\|\cdot\|$ to denote the corresponding induced matrix norm.

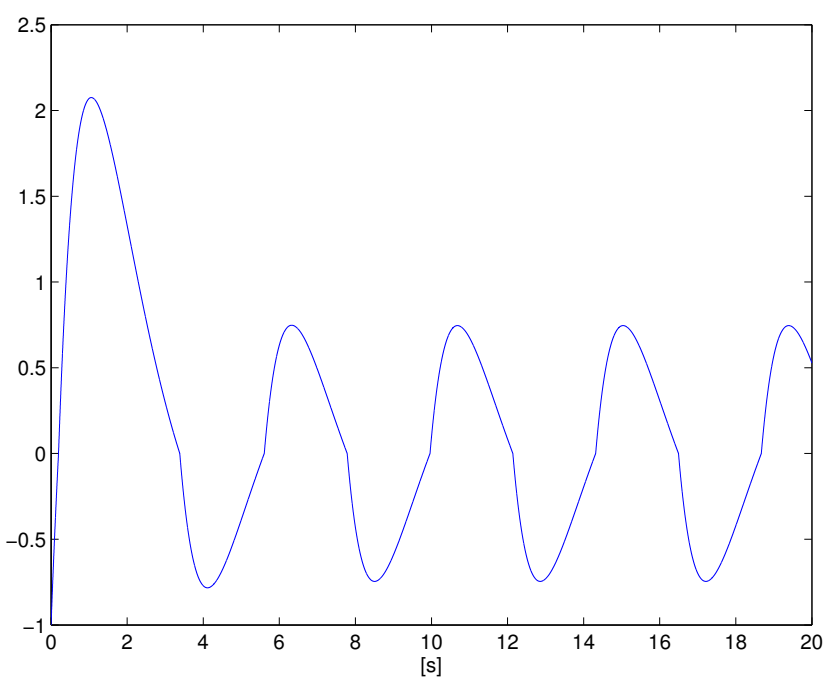

Figure 2: Output $-c x$ of the relay feedback system (1) with (3) but without dither signal $(\delta \equiv 0)$.

\section{A Motivating Example}

A second-order relay feedback system is used as a representative example. Consider the system (1) with

$$
L=\left[\begin{array}{cc}
-2 & -1 \\
1 & 0
\end{array}\right], \quad b=\left[\begin{array}{l}
1 \\
0
\end{array}\right], \quad c=\left[\begin{array}{ll}
1 & -1
\end{array}\right] \text {. }
$$

The linear part of the relay feedback system thus has a nonminimum-phase zero at 1 and a double pole at -1 . When no dither is present $(\delta(t) \equiv 0)$, the relay feedback system presents a limit cycle as reported in Figure 2. The output of the linear part $-c x$ of (1) is plotted for a solution with initial condition $x_{0}=\left[\begin{array}{ll}2 & 1\end{array}\right]^{T}$. If we apply a triangle dither signal $\delta$ with amplitude $A=1$ and period $p=1 / 50$, the limit cycle in Figure 2 is dissolved as shown in Figure 3. Hence, the dither in a sense attenuates the oscillations present in the original system. Figure 3 shows also the output $-c w$ of the smoothed system (2). The two systems have almost identical responses. Hence the smoothed system provides an accurate approximation of the dithered system for $p=1 / 50$. Figure 4 shows the responses when the dither signal has a larger period: $p=1$. The responses are no longer close and the output of the dithered system (solid) is oscillating with a larger ripple.

The simulations suggest that the dither period $p$ is related to how accurately the smoothed system approximates the dithered system. In next section it is shown that by choosing $p$ sufficiently small the approximation can be made arbitrarily tight (Theorem 4.1). Regarding the dither amplitude $A$, note that the smoothed system is not absolutely stable for $A<1 / 2$, since the closed-loop system is linear with characteristic polynomial equal to $s^{2}+\left(2-A^{-1}\right) s+1+A^{-1}$ when $|c w|<A$. The dither amplitude hence defines the response dynamics. This is shown in next section by relating $A$ to the stability of the dithered system (Theorem 4.2). 


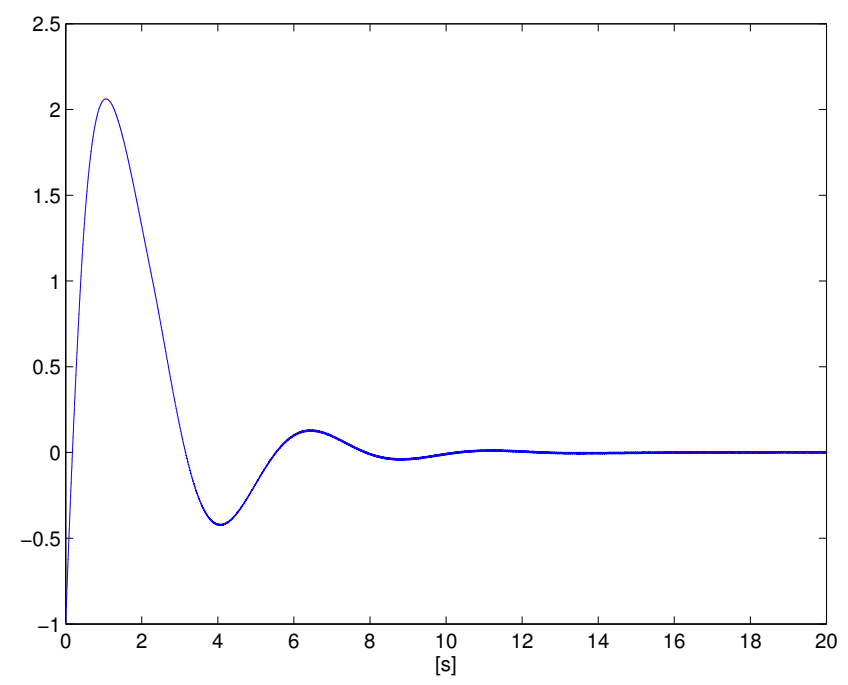

Figure 3: Outputs of the dithered relay feedback system (1) (solid) and the smoothed system (2) (dashed). The responses are almost identical.

\section{Main Results}

This section presents two result for the dithered system: one on accurate approximation and one on practical stability.

\subsection{Accurate Approximation}

The following theorem states that by choosing the dither period $p$ sufficiently small, it is possible to make the solution $x$ of the relay feedback system arbitrarily close to the solution $w$ of the smoothed system on any compact time interval.

Theorem 4.1 Let $T, \varepsilon>0$ and $x_{0} \in \mathbb{R}^{n}$ be given. There exists $p_{0}>0$ such that if $p \in\left(0, p_{0}\right)$, then $\left|x\left(t, x_{0}\right)-w\left(t, x_{0}\right)\right| \leq$ $\varepsilon$ for all $t \in[0, T]$.

Proof: Here we provide a sketch of the proof (the complete proof is reported in [15]).

Consider the dithered system (1) and the smoothed system (2) on the time interval $[0, T]$ and with $w(0)=x(0)=x_{0}$.

Note that the right-hand side of (1) is bounded on every compact time interval $[0, T]$, so there exists a positive constant $M$ such that $|c \dot{x}(t)| \leq M$, for all $t \in[0, T]$. An explicit estimate for $M$ is given below. By integrating the two members of (1)-(2), we obtain

$$
\begin{aligned}
x(t)-w(t)= & L \int_{0}^{t}[x(s)-w(s)] d s \\
& +b \int_{0}^{t}[n(c x(s)+\delta(s))-N(c w(s))] d s .
\end{aligned}
$$

The idea is to show that $\int_{0}^{t}[n(c x(s)+\delta(s))] d s$ can be approximated as $\int_{0}^{t} N(c x(s)) d s$ and the error introduced by this approximation is a function of the dither period $p$ and it can

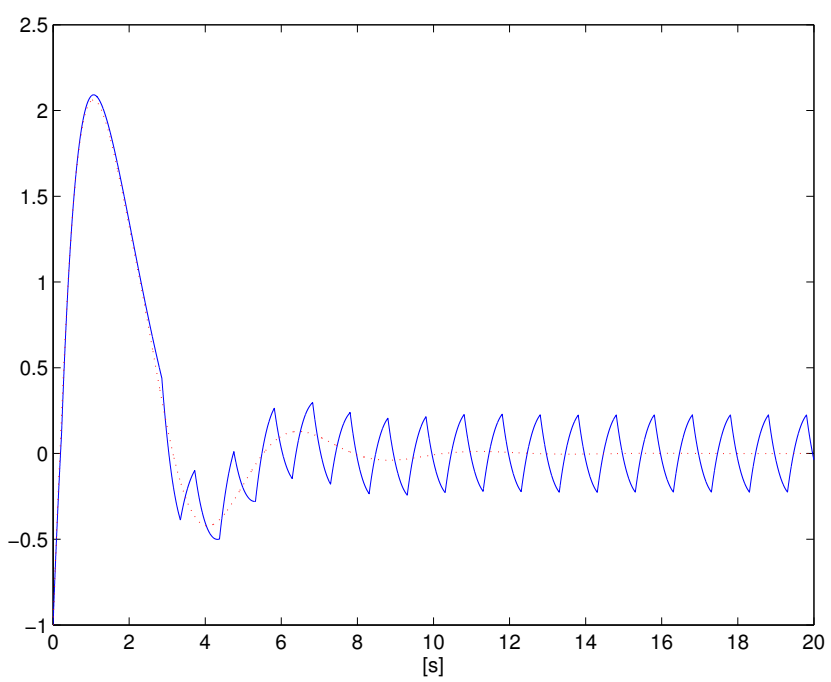

Figure 4: Outputs of the dithered relay feedback system (1) (solid) and the smoothed system (2) (dashed). Similar simulation as in Figure 3 but with dither signal having 50 times longer period. Note the deviation between the responses.

be made little by decreasing the period $p$. This is not obvious, particularly, since $n$ is a discontinuous nonlinearity. It is shown in [15] that (4) can be written as

$$
\begin{aligned}
x(t)-w(t)= & L \int_{0}^{t}[x(s)-w(s)] d s \\
& +b \int_{0}^{t}[N(c x(s))-N(c w(s))] d s+O(p),
\end{aligned}
$$

where $O(p)$ is the approximation error of ordo $p$. Then, by using the Lipschitz property of the nonlinearity $N$ and by applying the Grönvall-Bellman Lemma [18], we get

$$
|x(t)-w(t)| \leq O(p) e^{\left(\|L\|+\frac{|b \cdot| c \mid}{A}\right) T}=\varepsilon, \forall t \in[0, T] .
$$

An estimate of $p_{0}$ of the theorem can be derived:

$$
p_{0}=\min \left(\frac{4 A}{7 M}, \frac{\varepsilon}{\left(\frac{9}{2} \cdot \frac{M}{A} T+2\right)|b| e^{\left(\|L\|+\frac{|b| \cdot|c|}{A}\right) T}}\right) .
$$

Note that the smoothed system (2) has a bounded solution $w(t)$ on a finite time interval $[0, T]$, so there exists $d>0$ such that $|w(t)| \leq d$, for all $t \in[0, T]$. A possible choice of $M$ (the bound of $|c \dot{x}|$ ) based on (1) and (7) is

$$
M=\|L\|(d+\varepsilon)+\frac{|b| \cdot|c|}{A} .
$$

The bound obtained for $p_{0}$ in the proof is conservative, since the derivation is done using no particular knowledge of the 
system data. Tighter bounds can be obtained by exploiting more of the problem structure [16], but is not needed for the proof of the theorem.

Theorem 4.1 can be interpreted as an extension of Theorem 1 in [3] to a class of nonsmooth systems. The result in [3] relies on continuity properties of the solutions of the original and the smoothed systems. This argument cannot be used here, since a relay feedback system in general do not have solutions that depend continuously on initial conditions or system parameters. Instead, we pay particular attention in the proof to the system evolution at and between relay switchings.

\subsection{Practical Stability}

We will use Theorem 4.1 to obtain conditions for practical stability of the dithered system (1). The idea is the following. First we choose the amplitude $A$ of the dither signal, such that the smoothed system in (2) is stable. Then if the period $p$ of the dither signal is chosen small enough, the output of the dithered system closely follows the output of the smoothed system. This implies that the output of the dithered system converges close to zero. Note that we cannot obtain convergence strictly to zero, since the dither signal always cause small fluctuations of the output. We use the following definition of stability.

Definition 4.1 (Practical stability) The system in (1) is called practically (exponentially) stable if for any $\varepsilon>0$ there exists $A>0, p_{0}>0, \alpha>0$, and $\beta \geq 1$, such that

$$
|x(t)| \leq \beta e^{-\alpha t}\left|x_{0}\right|+\varepsilon, \quad \forall t \in[0, \infty)
$$

for any dither period $p \in\left(0, p_{0}\right)$.

There are many available results for stability analysis of the smoothed system. We will here use a criterion by Zames and Falb [17], which generalizes the Popov criterion.

Theorem 4.2 Let $G(j \omega)=-c(j \omega I-L)^{-1} b$ with L Hurwitz and consider some $H(j \omega)=\int_{-\infty}^{\infty} h(t) e^{-j \omega t} d t$, where $h: \mathbb{R} \rightarrow$ $\mathbb{R}$ satisfies $\int_{-\infty}^{\infty}|h(t)| d t \leq 1$. If there exists $\varepsilon>0$ such that

$$
\operatorname{Re}(G(j \omega)+A)(1+H(j \omega)) \geq \varepsilon, \forall \omega \in \mathbb{R},
$$

then there exists $p_{0}$ such that for $p \in\left(0, p_{0}\right)$ the system (1) is practically stable.

\section{Proof: See [15].}

Note that the criterion (9) corresponds to one of the least conservative conditions for stability available for systems with a slope restricted nonlinearity. However, it does not give any immediate information on the performance (e.g., the exponential decay parameters), and it is not convex in the pair $A, H$. The most straightforward use of the theorem is to put $H=0$, which corresponds to the circle criterion.
From the Kalman-Yakubovich-Popov Lemma one can then derive a linear matrix inequality that verifies (9) and results in explicit estimates of the exponential decay parameters, $\alpha_{0}, \beta_{0}$, for the smoothed system.

\section{Dither Design}

In this section we use Theorems 4.1 and 4.2 to design the dither signal, for example, in order to stabilize an oscillation. We also present a heuristic method, which gives less conservative designs. The design methods are illustrated on the example in Section 3.

\subsection{Tuning Algorithm}

The dither design choice will necessarily be a compromise between conflicting consequences of the dither amplitude $A$ and dither period $p$ on the control performance. Based on our theoretical results we obtain the following algorithm for tuning the parameters of the dither signal.

Step 1 Choose $A$ based on (9) in Theorem 4.2, so that the smoothed system in (2) is exponentially stable.

Step 2 Estimate $\alpha_{0}, \beta_{0}$ and let $T=-\ln \left(0.1 / \beta_{0}\right) / \alpha_{0}$, where $\alpha_{0}, \beta_{0}$ are the exponential stability parameters for the smoothed system.

Step 3 Choose $p_{0}$ based on $T$ and the smoothed dynamics.

A few comments are in place. In Step 1 we need to choose the amplitude $A$ of the dither signal large enough to allow the smoothed system to be stable and to have fast enough exponential decay rate. At the same time we want to keep $A$ as small as possible in order to avoid injecting a large signal in the control loop.

In Step 2 the estimates of $\alpha_{0}$ and $\beta_{0}$ can be derived based on the Kalman-Yakubovich-Popov Lemma, as discussed above. Then we can compute time interval length $T$, which is an auxiliary variable in the proof of Theorem 4.2. The parameter $T$ gives a bound on the period of the dither signal through (7) in the proof of Theorem 4.1. The bound is in general quite conservative, since it is derived without using any structure of the problem. It may thus suggest periods $p$ that are too small to be used in practice. Better bounds can be derived if we for example use the structure of the saturation nonlinearity and that the smoothed dynamics is chosen to be exponentially stable.

\subsection{Heuristic Tuning Rules}

A practical issue that can be taken into account when tuning the dither period is how much fluctuations on the output we get due to the dither signal. We derive a heuristic bound on these fluctuations.

Assume the transients have decayed and signals are small enough, so that we can consider the linear range of the 


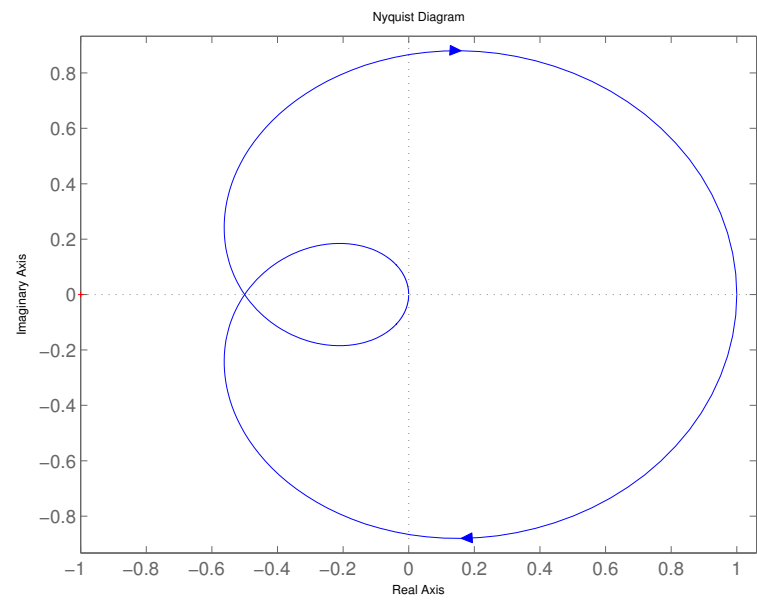

Figure 5: Nyquist curve of $G(s)=(1-s)(s+1)^{-2}$.

smoothed nonlinearity. Then the transfer function

$$
G_{\mathrm{cl}}(s)=\left(1+\frac{G(s)}{A}\right)^{-1} \frac{G(s)}{A},
$$

where $G(s)=-c(s I-L)^{-1} b$, approximately describe the mapping from the dither signal to the output $y=-c x$. Choose $\omega_{0}>0$ such that

$$
\left|G_{\mathrm{cl}}(j \omega)\right| \leq \frac{\mu}{A}, \quad \forall \omega \geq \omega_{0},
$$

for some small $\mu>0$. Then we can expect $|y(t)| \leq \mu$ for sufficiently large $t$, if the dither period is chosen such that $p \leq 2 \pi / \omega_{0}$. The following heuristic tuning rule follows:

Step 1 Choose an output bound $\mu>0$.

Step 2 Choose $A$ such that the circle criterion holds.

Step 3 Choose $p$ such that $p \leq 2 \pi / \omega_{0}$, where $\omega_{0}$ satisfies (10).

\subsection{Example Revisited}

Let continue discussing the example in Section 3. Recall that

$$
G(s)=-c(s I-L)^{-1} b=\frac{1-s}{(s+1)^{2}} .
$$

Consider Theorem 4.2 with $H(s)=0$, which corresponds to the circle criterion. We see from the Nyquist curve of $G$ in Figure 5 that for $A>0.56$

$$
\operatorname{Re} G(j \omega)+A \geq \varepsilon, \forall \omega \in \mathbb{R} .
$$

Hence, the dithered system is practically stable for $A>0.56$ and $p$ sufficiently small. By using Theorem 4.2 instead with $H(s)=-(s+1)^{-1}$, we can prove practical stability for $A>0.501$. Figure 6 shows a simulation for $A=0.502$ and $p=1 / 10$. It is clear that the system is close to the stability boundary for this choice of $A$.

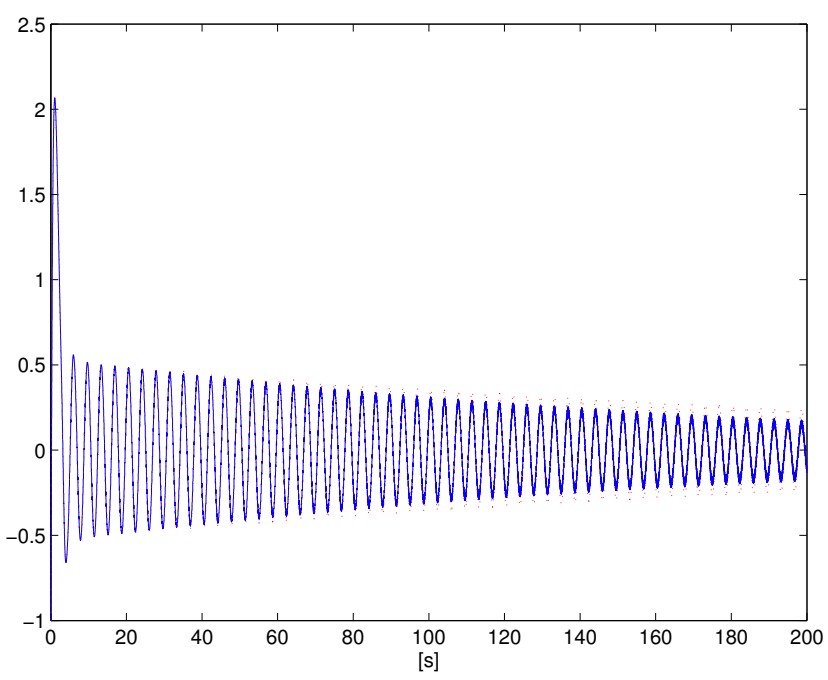

Figure 6: Outputs of the dithered (solid) and smoothed (dotted) systems close to the stability boundary predicted by Theorem 4.2.

Figure 7 shows the effect of the dither amplitude on the stability of the smoothed system: it is possible to obtain a fast convergence by increasing $A$. The upper plot shows a simulation for $A=0.56$ and the lower $A=0.70$.

Figure 8 shows finally the effect of the dither frequency on the approximation between the dithered system and the smoothed system: it is possible to obtain a response very close to the output of the smoothed system by decreasing the dither period. Compare the figure in Section 3.

\section{Conclusions}

In this paper we have shown how dither can be analyzed in nonsmooth systems. The main result is that a relay feedback system with a triangular dither signal at the input of the hard nonlinearity can be viewed as a feedback system (without dither) in which the relay is replaced by a saturation. While the amplitude of the dither signal affects the slope of the saturation, the approximate equivalence between the dithered and smoothed systems depends on the frequency of the dither signal. Explicit relations to achieve a desired approximation error have been given. Furthermore analytical and practical guidelines to design dithered systems have been presented. They were verified by simulations.

These preliminary results are the basis for some going work on the bound computation improvement, analysis of dither signals with different shapes, infinite time horizon extension of the Theorem 4.1 [16]. 

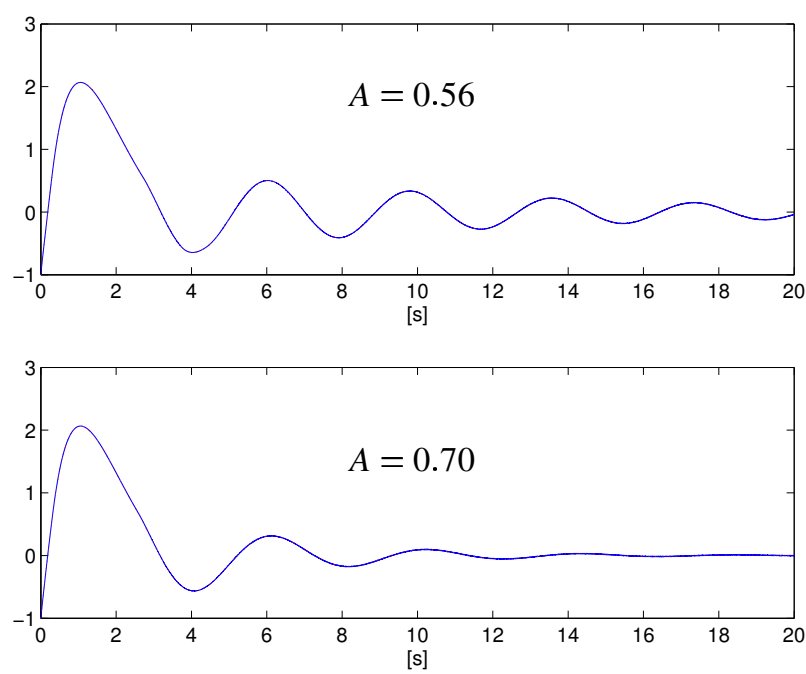

Figure 7: Output of the dithered system with $\delta$ having period $p=1 / 50$. The amplitude is $A=0.56$ (upper) and $A=$ 0.7 (lower), respectively. A smaller $A$ gives thus a less oscillating response.

\section{References}

[1] G. Zames and N. A. Shneydor, "Dither in non-linear systems", IEEE Transactions on Automatic Control, vol. 21, no. 5, pp. 660-667, October 1976.

[2] G. Zames and N.A. Shneydor, "Structural stabilization and quenching by dither in non-linear systems", IEEE Transactions on Automatic Control, vol. 22, no. 3, pp. 352361, June 1977.

[3] S. Mossaheb, "Application of a method of averaging to the study of dither in non-linear systems", International Journal of Control, vol. 38, no. 3, pp. 557-576, September 1983.

[4] K.J. Åström and B. Wittenmark, Adaptive Control, Addison-Wesley, Reading, MA, 1989.

[5] B. Armstrong-Helouvry, Control of Machines with Friction, Kluwer Academic Publisher, Boston, 1991.

[6] B. Armstrong-Helouvry, P. Dupont, and C. Canudas de Wit, "A survey of models, analysis tools and compensation methods for control of machines with friction", Automatica, vol. 30, no. 7, pp. 1083-1138, 1994.

[7] A. V. Peterchev and S. R. Sanders, "Quantization resolution and limit cycling in digitally controlled PWM converters", in Proc. IEEE Power Electronics Specialists Conf., Vancouver, Canada, June 2001.

[8] R.M. Gray and D.L. Neuhoff, "Quantization", IEEE Transactions on Information Theory, vol. 44, no. 6, pp. 2325-2383, 1998.

[9] Ya. Z. Tsypkin, Relay Control Systems, Cambridge University Press, Cambridge, UK, 1984.

[10] V.I. Utkin, Sliding Modes in Control and Optimization, Springer Verlag, Berlin, 1992.
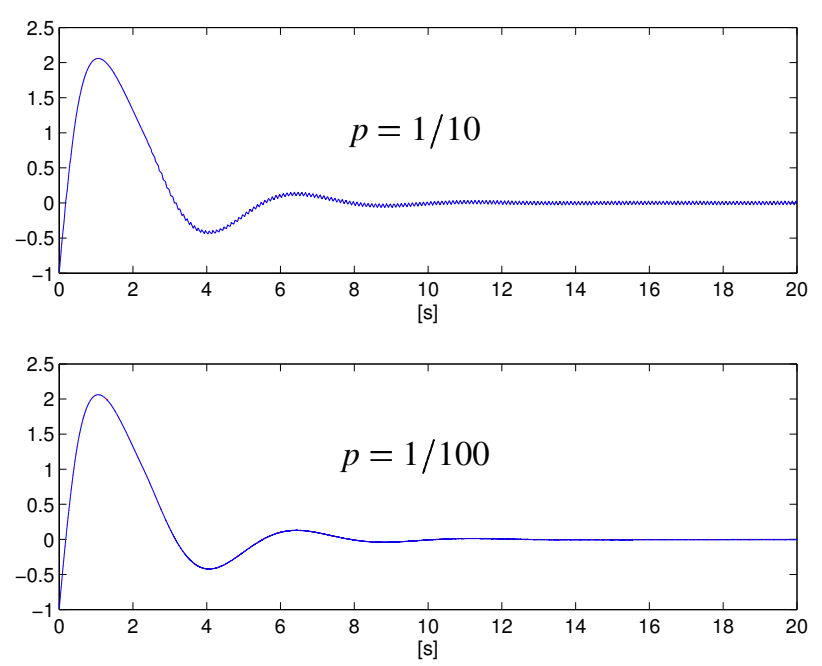

Figure 8: Output of the dithered system with $\delta$ having amplitude $A=1$. The period is $p=1 / 10$ (upper) and $p=1 / 100$ (lower), respectively. A smaller $p$ gives a better agreement between the responses of the dithered and smoothed systems.

[11] A. A. Pervozvanski and C. Canudas de Wit, "Asymptotic analysis of the dither effect in systems with friction", Automatica, vol. 38, no. 1, pp. 105-113, January 2002.

[12] D. Atherton, Nonlinear Control Engineering, Van Nostrand Reinhold Co., London, 1975.

[13] B. Lehman and R. Bass, "Extensions of averaging theory to power electronics", IEEE Transactions on Power Electronics, vol. 11, no. 4, pp. 542-553, 1996.

[14] A. Kh. Gelig and A. Churilov, Stability and Oscillations of Nonlinear Pulse Modulated Systems, Birkhäuser, Berlin, 1998.

[15] L. Iannelli, K.H. Johansson, U. Jönsson, and F. Vasca, "Analysis of dither in relay feedback systems", Tech. Rep., Dept. of Signals, Sensors and Systems, Royal Institute of Technology, Stockholm, Sweden, March 2002.

[16] L. Iannelli, K.H. Johansson, U. Jönsson, and F. Vasca, "Dither for smoothing relay feedback systems: an averaging approach", manuscript in preparation, 2002.

[17] G. Zames and P.L. Falb, "Stability conditions for systems with monotone and slope-restricted nonlinearities", SIAM Journal of Control, vol. 6, no. 1, pp. 89-108, 1968.

[18] S. Sastry, Nonlinear Systems: Analysis, Stability and Control, Springer-Verlag, New York, 1999. 